\title{
Elastic, Geo-Distributed RAFT
}

\author{
Zichen $\mathrm{Xu}$ \\ Generic Operational and Optimal Data \\ Lab, The Nanchang University \\ Nanchang, Jiangxi, China \\ xuz@ncu.edu.cn
}

\author{
Christopher Stewart \\ Computer Science and Engineering, \\ The Ohio State University \\ Columbus, Ohio, USA \\ cstewart@cse.ohio-state.edu
}

\author{
Jiacheng Huang \\ Generic Operational and Optimal Data \\ Lab, The Nanchang University \\ Nanchang, Jiangxi, China \\ jiachenghuang@email.ncu.edu.cn
}

\begin{abstract}
Raft is a protocol to maintain strong consistency across data replicas in cloud. It is widely used, especially by workloads that span geographically distributed sites. As these workloads grow, Raft's costs should grow, as least proportionally. However, auto scaling approaches for Raft inflate costs by provisioning at all sites when one site exhausts its local resources. This paper presents Geo-Raft, a scale-out mechanism that enables precise auto scaling for Raft. Geo-Raft extends Raft with the following abstractions: (1) secretaries which takes log processing for the leader and (2) observers which process read requests for followers. These abstractions are stateless, allowing for elastic auto scaling, even on unreliable spot instances. Geo-Raft provably preserves strong consistency guarantees provided by Raft. We implemented and evaluated Geo-Raft with multiple auto scaling techniques on Amazon EC2. Geo-Raft scales in resource footprint increments 5-7X smaller than Multi-Raft, the state of the art. Using spot instances, Geo-Raft reduces costs by $84.5 \%$ compared to Multi-Raft. Geo-Raft improves goodput of 95thpercentile SLO by 9X.Geo-Raft operates key-value services for 6 months without losing data or crash.
\end{abstract}

\section{INTRODUCTION}

Raft is a networked service that supports strong consistency. Distributed systems use Raft to reach consensus between software components. For example, components must agree on locking ownership and elements in synchronized queues. Raft is used in practice by large-scale platforms, such as Google Kubernetes [6], Core OS [4], and Oracle [5]. These platforms, i.e., the clients for Raft, suffer inflated costs when Raft employs inefficient scaling techniques.

Software threads in Raft are either leaders or followers. At all times, Raft allows zero or one leader as elected by followers. Followers periodically message the leader to check for failures. Upon leader failure, followers call for an election to name a new leader thread. The leader has a more demanding workload: it pushes writes to all available followers and tracks which followers have confirmed the most recent writes. The leader bottlenecks Raft throughput.

When workload demands overwhelm the leader, Raft infrastructure must acquire and use new resources to improve its throughput. One approach is to run the leader on more powerful computers (scale

Permission to make digital or hard copies of all or part of this work for personal or classroom use is granted without fee provided that copies are not made or distributed for profit or commercial advantage and that copies bear this notice and the full citation on the first page. Copyrights for components of this work owned by others than the author(s) must be honored. Abstracting with credit is permitted. To copy otherwise, or republish, to post on servers or to redistribute to lists, requires prior specific permission and/or a fee. Request permissions from permissions@ acm.org.

IWQoS '19, June 24-25, 2019, Phoenix, AZ, USA

(C) 2019 Copyright held by the owner/author(s). Publication rights licensed to ACM. ACM ISBN 978-1-4503-6778-3/19/06 . .\$15.00

https://doi.org/10.1145/3326285.3329046 up). When more powerful computers are unavailable, the leader's load must be split (scale out). Herein lies the problem: Raft permits one leader only. One leader is essential to make the algorithm understandable, correct, and easy to implement. Multi-Raft [16] scales out by replicating leaders and followers, and splitting data between replicas. Each replica implements a Raft, providing strong consistency. Between replicas, 2 phase commit provides strong consistency. Multi-Raft can improve throughput as workload demand increases, but it is costly. Each scale out operation doubles resource footprint.

With the global proliferation of networked mobile devices, follower nodes in Raft are increasingly geo-distributed. Consider global, coordinated release of video content, Raft coordinates when such content is accessible. Placing followers in geo-distributed data centers ensures low latency access times for all users. The cost of cloud resources needed to run followers varies from site to site. By naïvely replicating leaders and followers, the Multi-Raft approach scales out at expensive sites. Our research seeks a solution that selectively excludes expensive sites during scale out, without compromising throughput or latency.

Multi-leader Paxos algorithms allow multiple software threads to process writes. These algorithms do not suffer follower-leader workload imbalance like Raft. However, these algorithms require complicated procedures to handle partitions, add software threads, and remove threads. Subtle programming mistakes can invalidate strong consistency guarantees, leading to costly bugs. Raft allows a single leader only by design, simplifying programming for Raft. Such simplicity is a key feature of Raft that must be preserved.

We present Geo-Raft, an extension that scales well with geodistributed resources. Geo-Raft supports 4 types of software threads: followers, leaders, secretaries and observers. Like original Raft, GeoRaft permits zero or one leader based on majority votes by followers. In Geo-Raft, leaders outsource a portion of their workload to secretaries, reducing workload imbalance. Observers answer read-only queries, reducing workloads for followers. Geo-Raft allows multiple secretaries and observers to run simultaneously. Geo-Raft preserves the safety guarantees of Raft. We employ state irrelevancy [20] to prove that secretary and observer failures do not affect correctness.

Secretaries and observers are stateless and execute at any geodistributed site. They can be deployed incrementally to achieve high throughput at low cost as client workloads grow. We present a cost modeling approach to discover high throughput, low latency and low cost settings for Geo-Raft. Our approach accepts time sensitive parameters on cost and latency. This allows Geo-Raft to use geodistributed resources on spot markets, i.e., cloud markets with cheap but failure prone resources. For example, in Amazon AWS EC2, spot instances can cost $90 \%$ less than on-demand instances [24, 25, 34, 35]. Geo-Raft uses safe, on-demand instances for leaders and followers, and spot instances for secretaries and observers. In the 


\begin{tabular}{|c|c|c|c|c|c|c|c|c|c|c|c|c|c|c|c|}
\hline \multicolumn{16}{|c|}{ Write Operation } \\
\hline \multirow{2}{*}{\multicolumn{4}{|c|}{$\begin{array}{l}\text { client query:write } \\
\text { key A to value } 3\end{array}$}} & \multicolumn{3}{|c|}{ append leader log } & & owe & $\mathrm{fl}$ Ic & & \multirow{2}{*}{\multicolumn{4}{|c|}{$\begin{array}{c}\log \text { matching \& } \\
\text { follower log append }\end{array}$}} & \multirow{6}{*}{$\begin{array}{c}\text { follower } \\
\text { confirmation } \\
\text { commit hash } H \\
\mathrm{H}(1,6) \rightarrow \mathrm{H}(1,6)+\mathrm{f} 1\end{array}$} \\
\hline & & & & term id & key & val & term & & key & val & & & & & \\
\hline & & \multirow[t]{2}{*}{ A } & \multirow[t]{4}{*}{3} & \multirow{2}{*}{\begin{tabular}{|l|}
0 \\
0 \\
\end{tabular}} & \multirow{2}{*}{\begin{tabular}{l|l}
$A$ & \\
$B$ & \\
\end{tabular}} & & 0 & 3 & A & 2 & \multirow[t]{2}{*}{0} & \multirow[t]{2}{*}{3} & \multirow{2}{*}{ A } & & \\
\hline & & & & & & & 0 & 5 & C & & & & & & \\
\hline & & & & & & & & & & & 0 & 4 & B & \multirow{2}{*}{13} & \\
\hline & & & & 6 & $A$ & 3 & & & & & 1 & 6 & A & & \\
\hline \multicolumn{16}{|c|}{ Geo-distributed Scale Out } \\
\hline \multirow{2}{*}{\multicolumn{4}{|c|}{$\begin{array}{c}\text { partition leader log } \\
\text { raft \#1 }\end{array}$}} & \multirow{2}{*}{\multicolumn{3}{|c|}{$\begin{array}{l}\text { partition follower logs } \\
\text { raft \#1 }\end{array}$}} & \multicolumn{9}{|c|}{ provision new instances and migrate } \\
\hline & & & & & & & & @eu & I-fran & kfurt & $@ a$ & sia-s & inga & ore & @us-east \\
\hline 0 & 3 & $A$ & 2 & 0 & 3 & 2 & & & & & & & & & \\
\hline 1 & 6 & $\mathrm{~A}$ & 3 & 1 & 6 & 3 & & & & & & & & & 2) \\
\hline \multicolumn{4}{|c|}{ raft \#2 } & \multicolumn{3}{|c|}{ raft \#2 } & & & & & & & & & \\
\hline 0 & 4 & $\mathrm{~B}$ & 1 & 0 & 4 & 1 & & & & & & & & & \\
\hline
\end{tabular}

Figure 1: Execution of writes in Raft (top) and geo-distributed scale out in Multi-Raft (bottom).

geo-distributed setting, Geo-Raft is cheap per se, as it leases spot instances from the cheapest market- not necessarily the market running the leader thread.

We deployed Geo-Raft on Amazon EC2 for 6 months serving key-value lookup based on Google traces [3]. Geo-Raft purchases low-cost spot instances in geo-distributed sites to scale-out the performance within the budget, and boosts both read and write throughput, compared to Raft [26]. Geo-Raft adaptively boosts read and write throughput which incurs less than $85 \%$ overhead compared to Raft. Geo-Raft scales in increments 5-7X smaller than Multi-Raft, the state of the art. Geo-Raft reduces costs by $84.5 \%$ compared to MultiRaft and improves goodput (i.e., the application-level throughput) of $95^{\text {th }}$ percentile SLO by 9 X.Geo-Raft operates key-value services for 6 months without losing data or crash.

Contributions are listed below:

- We present Geo-Raft, an extension to the widely used Raft algorithm that can use cheap spot instances.

- We use state irrelevancy to prove that Geo-Raft preserves key features of Raft.

- We present a cost modeling approach to manage cloud instances for Geo-Raft.

- We built Geo-Raft and deployed it for 9 weeks. Geo-Raft reduces costs by $84.5 \%$ compared to Multi-Raft.

- We used real systems to evaluate Geo-Raft, showing that offload can proceed quickly enough to speedup consensus protocols.

The remainder of this paper is organized as follows. Section 2 overviews Raft. Section 3 describes Geo-Raft and proves that it retains safety guarantees of Raft. Section 4 describes Geo-Raft implementation over geo-distributed spot markets. Section 5 illustrates the geo-raft can greatly reduce the cost. Section 6 provides a brief on raft algorithm and related work. At last, Section 7 concludes the paper.

\section{BACKGROUND}

Raft is commonly realized as a networked key-value store, supporting the following queries:
- revision id $\leftarrow$ write(key $\mathrm{k}$, value $\mathrm{v}$ )

- $\{$ value $\mathrm{v}$, revision id $\} \leftarrow \operatorname{read}($ key $\mathrm{k})$

Raft sets a global processing order for write queries and ensures that subsequent read queries will return the same value or the value of a later write query. In other words, Raft supports linearizable consistency.

Figure 1 (top) depicts write query execution. The client provides key and value when issuing the query. The leader uses current state of its $\log$ to append term and revision id. Leader elections increment term. Write queries increment revision id.

Hardware failures and network partitions can make leader and follower logs diverge. However, Raft ensures that nodes always agree on one leader. As Figure 1 (top) shows, the leader gets logs from followers and align them with their own. Raft requires followers to accept updated logs from the leader. The process of aligning logs is called log matching.

In Raft, software threads are either the leader, followers or leader candidates. The leader maintains its role by sending heartbeat messages. After receiving a heartbeat, followers set a random timer. If they do not receive a message before the timer triggers, the follower calls for an election. The follower increments the term in its logs and tells other followers that it is a candidate for leader. Followers vote for a candidate whose $\log$ is not older than its own. If a candidate gets the votes of most nodes, it will become a new leader.

Scaling out with Raft: Raft permits only one leader at a time and the leader has a demanding workload. Naively adding follower nodes does not improve throughput. In fact, it often degrades throughput by causing more log matching.

Multi-Raft is a widely used approach to scale out. As shown in Figure 1 (bottom), it sets up multiple Raft services, splits the key space and assigns each split to one Raft. Multi-Raft then migrates each Raft service to its own resources. In geo-distributed cloud settings, each Raft service uses instances at each site. For example, Figure 1 shows migration to new instances at AWS EU-Frankfurt, Asia-Singapore and US-East sites. Each Raft is independent and handles their own update, log commit, and leader election (e.g., raft 


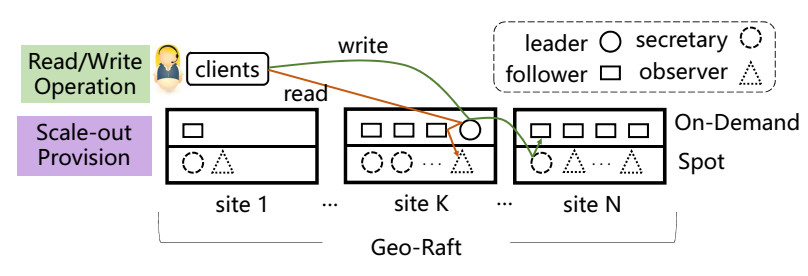

Figure 2: Geo-Raft scales out using spot market resources.

\#1 and raft \#2 in Figure 1). Between leaders of these Raft, they communicate and keep in consistency based on 2 Phase Commit.

The Multi-Raft approach preserves linearizability within key ranges and scales out well. However, it is not cost efficient. Scale-out can double the resource footprint to resolve light bottlenecks at only one leader nodes.

\section{DESIGN}

In this section, we describe the design of Geo-Raft. As aforementioned before in Section 1, Geo-Raft handles raft protocols over a list of sites to provide data services with strong consistency. Such performance is obtained using our Geo-Raft protocols. Geo-Raft operates original raft among sites while hiring secretary instances to take over the maintenance job from the leader instance. As such, we allow the leader to handle a much larger number of followers in a single raft model. Once the leader instance (i.e., on-demand instance) fails, the partial raft will be considered not a working duplicate of the data service.

Geo-Raft extends Raft with two new types of software threads: secretaries and observers. Secretaries relieve log analysis from the leader and observers relieve read requests from followers. Secretaries and observers are stateless, allowing for scale up and down.

Geo-Raft ensures linearizable consistency. Like Raft, Geo-Raft allows only one leader thread. Also, the leader is selected from followers via election. Secretaries and observers can not vote. As such, Geo-Raft can support any number of secretaries and observers. In this section, we will show that Geo-Raft provably maintains correctness guarantees.

Secretaries and observers can run on cheap, failure-prone resources, making them well suited for geographically distributed spot markets, i.e., cloud resources that are heavily discounted but can be revoked at any time. Figure 2 provides an overview of our GeoRaft. Clients issue write queries to the leader. The leader offloads log matching and related tasks to a secretary which runs on a spot instance. Read queries are handled by either followers or observers. Note, the number of secretaries and observers varies from site to site and fluctuates.

This section first details our algorithm, with a focus on leader election, secretary and observer failures, and the main safety guarantee. Then, we present a modeling approach for managing cloud resources cost effectively.

\subsection{The Geo-Raft Algorithm}

Geo-Raft, like the original Raft, initializes cloud instances at all sites and runs leader and follower software threads on them. Figure 3

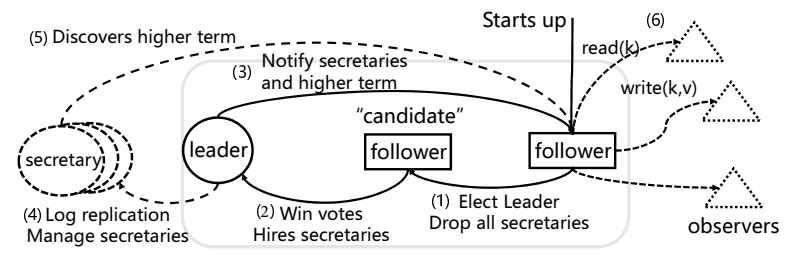

Figure 3: The state machine and stateless nodes in Geo-Raft. The central part in rectangle preserves the classic Raft state machine.

shows the whole state transfer in Geo-Raft, starting from leader election.

Geo-Raft Leader Election: The leader manages log matching and replication for followers and secretaries. The execution trace of commands for the state machine is based on logging.

PROPERTY 3.1. (Leader Election Safety). Like Raft, there is at most one leader per term in Geo-Raft.

The leader maintains its role by sending heartbeat messages. After receiving a heartbeat, followers set a random timer. If they do not receive a message before the timer triggers, the follower calls for an election and stops all secretary threads (i.e., Step (1) in Figure 3). The follower increments the term in its logs and tells other followers that it is a "candidate" for leader. Followers vote for a candidate whose $\log$ is not older than its own. If voting times out, the election will restart again (not shown in Figure 3). If a candidate/follower gets the majority votes from most followers, this node will become a new leader and Geo-Raft provisions secretaries for the new leader (i.e., Step (2) in Figure 3).

Geo-Raft starts each term $(\bar{T})$ with a leader election. The leader orchestrates normal operations (log management), notifies higher term when new update, as shown in Step (3) in Figure 3. Meanwhile, the leader in Geo-Raft always tells followers which secretaries are responsible for this log, such that log management can be offload to assigned secretaries (i.e., Step (5) in Figure 3). If the election is a failure, a new term starts, with a new election.

PROPERTY 3.2. (Geo-Raft State Machine Safety). Each replicated copy of the state machine executes the same commands in the same order.

Established in Woos and Wilcox's prior research in Raft [31], this property provides consistency guarantees for the logs between all leader, secretaries, and followers in the protocol.

Stateless Operations in Geo-Raft Leader Election: Besides the whole leader election process, Geo-Raft employs stateless threads as secretaries and observers. After a successful election, when the term $(\hat{T})$ is longer than a predefined period $(T<\hat{T})$, leader will reprovision secretaries and offload logs (i.e., Step (4) in Figure 3 ) by the end of this period. Geo-Raft adopts this re-election mechanism to mitigate the fail-prone feature from spot instances, without incurring noticeable delay/cost.

Followers that operate in Geo-Raft can arbitrarily hire cheap local spot instances as observers. Each time a follower receives a write, it 


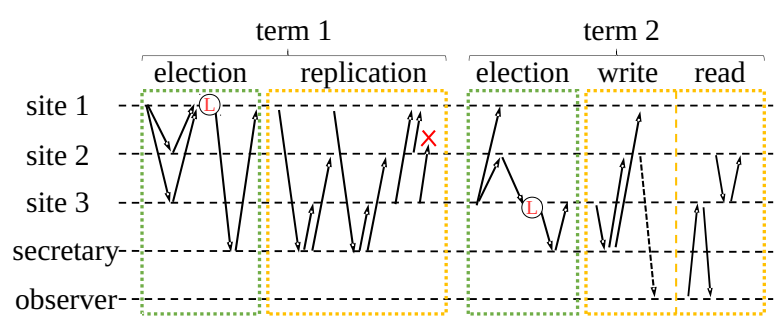

Figure 4: Operations in Geo-Raft.

will append this write to all its linked observers without waiting for the commit. When the follower receives a read, it reroute the job to committed observers. If no observers commit previous appending $\log$, this follower execute the read itself (i.e., Step (6) in Figure 3). Log Replication: During normal operation, the whole Geo-Raft is in the log replication phase. In log replication, the list of commands to execute on the state machine is kept in the log, and the position of a command in the log is called its index. Each node has its own copy of the $\log$, and state machine safety reduces to maintaining agreement between all copies of the log. Figure 4 shows an example of GeoRaft. When a client sends a write to the leader (e.g., an instance in Site 1), the leader first appends a new log entry containing that command to its local log. Then the leader sends an append message containing the entry to an assigned secretary, which is responsible to confirm with all other instances in Geo-Raft (i.e., leader offloads to secretary). Each other node appends the entries to its $\log$ (e.g., arrow from site 1 to site 2,3 during replication in Figure 4) before sending the acknowledgment to the assigned secretary (e.g., arrows from site 2,3 to site 1 during replication in Figure 4). To ensure that followers' logs stay in consistent with the log at the leader, messages include the index and term of the previous entry in secretary's log; the follower checks whether it has an entry with the index and term before appending new entries to its log. This consistency check guarantees the following property:

PROPERTY 3.3. (Log Matching). If any two logs contain entries at a particular index and term at different locations, then the logs are identical in Geo-Raft.

Once the secretary learns the majority followers have acknowledged the new entry, it will notify the leader and mark this write as committed. Note that, the leader need not receive commit from every single secretary before proceeding requests. The majority of secretary acknowledgment confirms the majority notes from followers. Then, the leader then executes the command contained in the committed entry on the state machine and responds to the client with the output of the command. The followers are informed that they can safely execute the command on their state machines, gossiping with its observers. Thus, in both leader election and log replication, both secretaries and observers are state irrelevant.

PROPERTY 3.4. (State Irrelevancy). If any secretaries/observers fail during the term at different locations, then the logs are still matched thus state machines in both leader and followers are irrelevant to secretaries and observers.
Proof. We first prove the State Irrelevancy in secretaries. Given an execution trace $\tau$ of Geo-Raft, assuming one secretary fails during the trace, we shall find an existing $\sigma$ such that $\tau$ linearizes to $\sigma$. To find the $\sigma$, we revert Geo-Raft back to a single Raft, then we pick the sequence of commands executed by the followers on their local state machines. State machine safety guarantees that all nodes agree on this sequence.

The remain job is to show that without failing certain secretaries, we have $\tau^{\prime}$ linearizes to $\sigma$. Let $\tau^{\prime}$ be the sequential inputâĂŞoutput trace corresponding to $\sigma$ in $\tau$. That is, for each command executed in $\sigma, \tau^{\prime}$ contains an input immediately followed by the corresponding output for that command. All commands from/to secretaries can be omitted in $\tau^{\prime}$, which makes it a permutation of $\tau$ that respects the ordering condition of properties. Each of these is established as a separate invariant by induction on the execution. The proof for observers are similar since they never affect the order of local state machine of the correspondent followers.

With all properties inherited from Raft, while the additional roles in Geo-Raft are state-irrelevant, we have,

Theorem 3.5. Properties 3.1-3.4 imply linearizability in Geo-Raft.

The proof trivially follows the linearizability proof in Raft [31]. Once an entry is committed, it becomes durable, in the sense that its effect will never be forgotten by Geo-Raft. In all, Geo-Raft operates $\log$ replication with linearizability.

PROPERTY 3.6. (Linearizability). Geo-Raft implements a linearizable state machine.

The proof trivially follows the linearizability proof in Raft [31]. Geo-Raft also provides a liveness guarantee: if there are sufficiently few failures, then the system will eventually process and respond to all client commands.

\subsection{Managing resources for Geo-Raft}

Leader elections disrupt normal query processing, causing processing times to deviate from standard distributions. These delays force clients to wait to acquire locks, affecting the rate at which they issue new queries. To manage resources, we model processing times and arrival rates with a G/GI/m model, i.e., a model that supports generic distribution of arrival rate and generic independent distribution for mean processing time. There are $m$ cloud instances to process queries. Allen-Cunneen approximates [8] individual response time for $\mathrm{GI} / \mathrm{G} / \mathrm{m}$ models. Equation 1 models mean response time $(\bar{t})$.

$$
\bar{t}=\frac{1}{\mu}+\frac{\rho}{\mu(1-\rho)}\left(\frac{C_{A}^{2}+C_{B}^{2}}{2}\right)+\frac{2 E[I]+\lambda E[I]^{2}}{2(1+\lambda E[I])}
$$

In this equation, mean processing time is $\frac{1}{\mu}$, mean query arrival rate is $\lambda$, mean utilization of a server is $\rho=\frac{\lambda}{\mu}, C_{A}^{2}$ and $C_{B}^{2}$ represents the squared coefficient of variation of request inter-arrival times and request sizes, respectively, and $E[I]$ is mean initial set-up time for processing the first query.

Given a set of $m$ instances, we separate them into $m_{l}$ and $m_{f}$ as leader and followers, using on-demand instances; $m_{s}$ and $m_{O}$ represent secretaries and observers, using spot instances. Thus, the total number of required instances is $m=m_{l}+m_{f}+m_{s}+m_{o}$. The 
estimated expense is in Equation (2).

$$
c=\Sigma c_{k} m_{\text {role }}+C_{0}
$$

where $c_{k}$ is the local unit price of selected instance in data center $k$, $m_{\text {role }}$ represents the aforementioned set of instances for different Raft roles, and $C_{0}$ is a linear cost function of networking based on the total number of instance $m$.

As shown in Equation 2, the total cost (c) of one instance provision to deploy Geo-Raft depends on the price of instances for each software thread $\left(c_{i}\right)$. The number of instances $\left(m_{i}\right)$ also affects cost. For example, leasing too many failure-prone spot instances can increase costs enough to negate price savings. Also, arbitrarily increase the frequency of adapting spot instances that increase risks of failure and thus violating SLOs. We model the total revenue as a weighted function of (1) expense to lease instances and (2) failure rate of picking certain instances. Equations (3)-(9) show the model:

$$
\begin{aligned}
& \operatorname{Max} R \\
& R \triangleq\left\{c_{1}, c_{2}, \ldots, c_{(m, n)}\right\} \\
& c_{i}=r_{j}^{a} \vartheta_{j} \\
& r_{j}=(1-\tau) \iota_{j} \cdot \rho+\tau \iota_{j} \cdot \rho(1-\phi) \\
& \vartheta_{j} \triangleq \frac{\zeta}{\ell_{j}^{k_{j}}} \\
& k_{j}= \begin{cases}1, & \zeta \leq \omega \\
\infty & \zeta>\omega\end{cases} \\
& \ell_{i}=\sum p_{j} T
\end{aligned}
$$

In these equations, $i$ indexes the $i^{t h}$ leasing period of combined instances and $j$ indexes the $j^{t h}$ instance. $R$ is the possible maximum revenue when examining a vector of possible selections, i.e., $\left\{c_{1}, c_{2}, \ldots, c_{(m, n)}\right\}$, where $m$ is the total number of selected instances and $n$ is the total number of instances available. $c_{j}$ is the total revenue from serving workloads from selection $j$. The total revenue is a nonlinear combination of financial profit $\left(\vartheta_{j}\right)$ and correspondent performance variable $\left(r_{j}\right)$. Parameters $a$ is the weight coefficient.

Performance variable $r_{i}$ captures performance from both ondemand and spot instances. $\tau$ is the ratio of hired spot instances in $m$ nodes. one decision of selecting $\iota_{j}, \rho$ is the provisioned utilization from Equation 1. $\phi$ captures the spot failure rate. The total $\operatorname{cost} \vartheta_{j}$ captures gaining from the possible SLO $(\zeta)$ while spending $\left(\ell_{j}\right)$ for leasing the $i^{t h}$ selection. Note that we use $k_{j}$ as the weight coefficient when SLO $\zeta$ violates certain threshold $\varpi$. Usually, we set $\varpi=99.999 \%$. In our current setup, we do not want any SLO violation in Geo-Raft . Thus, violating the threshold $₫$ can lead to infinitely large expense, thus a zero revenue. The parameter $\left(\ell_{i}\right)$ calculate the total expense from the $i^{t h}$ selection in the period $T$. For the ease of analysis, we set $T=1$ hour.

Geo-Raft creates secretaries for leader. When a new leader voted or secretaries dead in the previous epoch, Geo-Raft hires new secretaries in the new epoch. If one leader fails, secretaries will be discarded and could be rehired in next epoch. But flimsy nodes might reduce the performance of raft algorithm. Frequent re-election might cause availability issue. Raft and other consistency algorithms were shut out of the spot market. Hence, we carefully implement

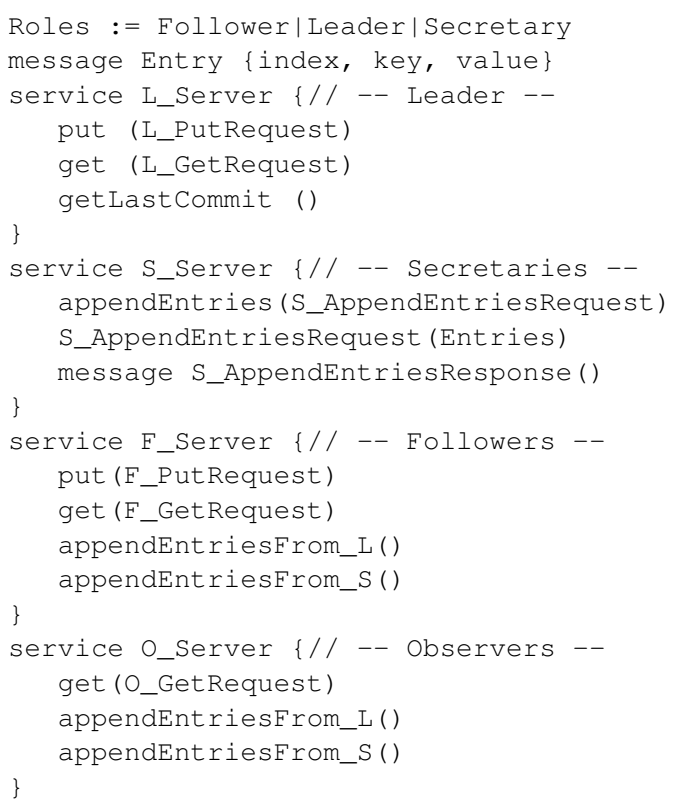

Listing 1: Remote Procedure Call in Geo-Raft

our Geo-Raft algorithm to improve throughput and reduce latency in distributed replication system by using spot instance.

\section{IMPLEMENTATION}

We prototyped Geo-Raft. The prototype consists of about three thousand lines in Java, which includes 4 kinds of thread roles, a storage, and a state machine. Leaders, followers, secretaries, and observers communicate via remote procedure calls (RPC).

Our prototype uses 4 types of RPC (shown in Listing 1). The first type of RPC allows leaders to start secretaries. In the second type, the leader uses RPC append follower logs. Recall from Figure 1, the data structure used to append entries includes $<$ term, index, key, value $>$. The leader also includes an address for an assigned secretary in this RPC. Followers reply to secretaries whom inform the leader when the log has been appended by a majority of followers. As in Raft, leader and follower RPC piggy backs heartbeat messages to maintain leadership. If there is no secretary in the runtime, leader directly replicates log entries to followers. In this case, Geo-Raft reverts and equals a classic Raft .

The third and forth RPC type occurs when secretaries offload log management and observers receives reads, respectively. Secretaries use RPC to discover matching indices within follower logs. Recall, secretaries are designed to run on cloud instances that could fail at any time. We hypothesize that future implementation of this RPC could use a highly frequent asynchronous communication. In our prototype, we used heartbeats in a short sliding window.

Geo-Raft realizes several interfaces which enable a scalable geodiverse replicated state machine. First, Geo-Raft provides an interface that implements strong consensus between sites. Among all sites, Geo-Raft leases global spot instances as secretaries for leaders. Within a site, Geo-Raft hires local spot instances as observers for 

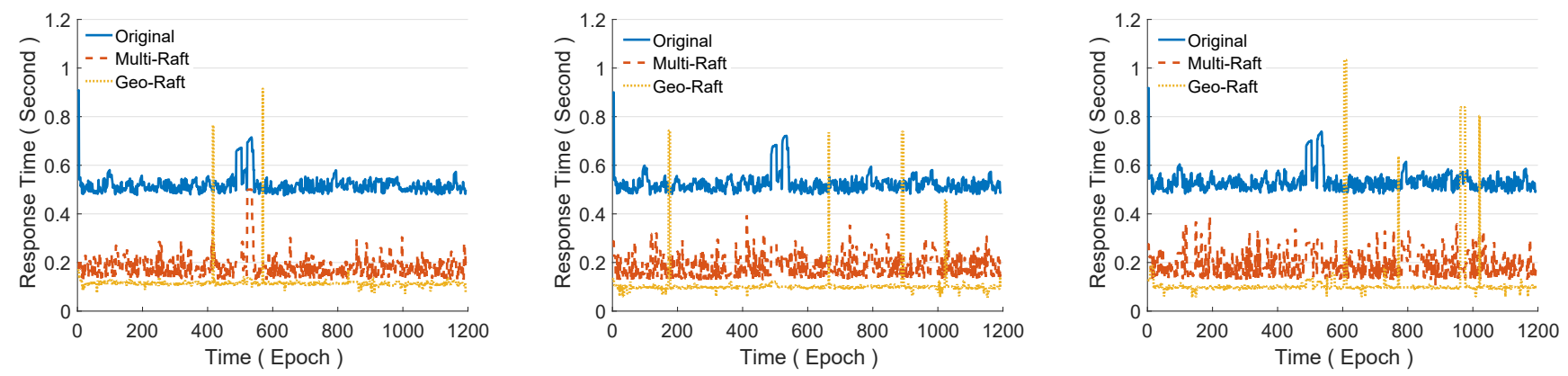

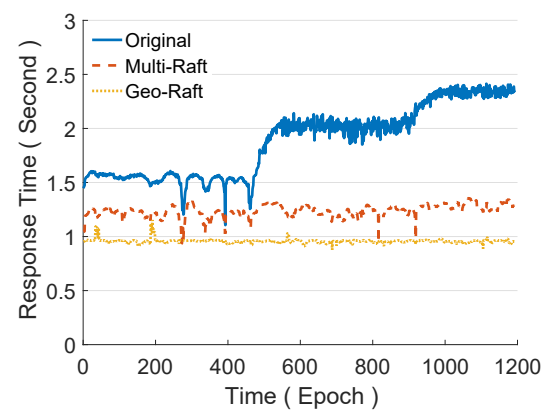

(a) Small

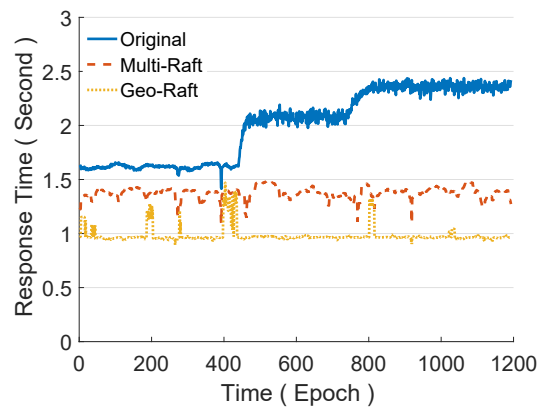

(b) Medium

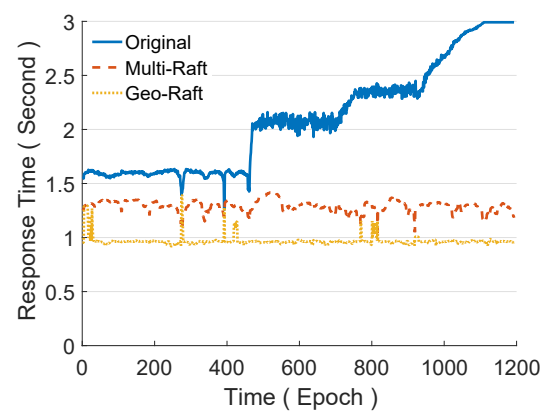

(c) Large

Figure 5: 50 days Performance snapshots running Read (top) and Write (bottom) workloads. Y axis is in log scale.

followers. The total number of spot instances is dynamically tuned based on current performance statistics and resource provision, as discussed in Section 3.2. Geo-Raft also exposes the management interface to clients, supporting upper-level applications. Hence, developers could switch to Geo-Raft with a few extra lines of codes.

Geo-Raft provides an interface to change the number of secretaries in a cluster. A suitable number of secretaries is depended on many factors such as message size, server capacity, followers number, workload pattern, server price, and etc. Different secretaries number might result in a large gap between same leader and followers nodes. Geo-Raft is a consistent protocol targeting global service. Secretary could be deployed in worldwide data centers. The location of secretary could be selected specially to get lower latency, lower network cost, and higher throughput.

\section{EVALUATION}

Geo-Raft is designed to maintain a cheap and scale-out raft protocol across on-demand and spot instances. In this section, we evaluate the performance of Geo-Raft by prototyping it onto our physical testbed and Amazon AWS EC2 [1, 23].

System Setup: We built a physical testbed to illustrate the runtime performance of Geo-Raft using Google workload. The testbed contains 96 nodes with Intel Xeon $26202.60 \mathrm{GHz}$ and 64GB DDR4 memory each. We also deploy Geo-Raft onto instances (t2.small) in Amazon AWS EC2. For the burstable spot instance, we report $0.415 \$ / \mathrm{H}$ on average. Spot instances were up to $90 \%$ cheaper than their regular on-demand instances. The average lifetime of a spot instance was 32 minutes and prices between sites were not correlated.
Software Setup: The operating system is built as CentOS 7 (kernel version 3.10.0). The experiments are built based on a client-server model, as shown in Figure 2. The client sends batched workloads as reads/writes, with different CPU/memory demand, arrived in a Poisson distribution.

In this paper, we mainly evaluate Geo-Raft with the following baselines:

- Original implements a state-of-the-art Raft design from Ongaro et al. [26];

- Multi-Raft is a state-of-the-art multi-raft implementation using sharding [15];

- Oracle is a baseline based on offline analysis.

Workloads and Traces: We verified the performance of Geo-Raft using real world workloads and traces. We use the popular Google cluster trace [3] which contains one-month job statistics in Google cluster. Workloads are random reads/writes, controlled ratio $\mathrm{R} / \mathrm{W}$ batches, and read/write-only workloads. All workloads are tested with small, medium, and large block sizes, namely $256 \mathrm{kB}, 1024 \mathrm{kB}$, and $2048 \mathrm{kB}$, respectively.

- Read is a batch of workloads with read-only queries.

- Write is a batch of workloads with all writes.

- $\alpha$-Static is an $\alpha$ controlled workload batch with $\alpha$ as the $\mathrm{read} / \mathrm{write}$ ratio.

\subsection{Results}

Performance Snapshots: First, we report the performance snapshot of running Geo-Raft, Multi-Raft, and Original. The whole experiment lasts for 1200 epochs (i.e., 50 days) in Amazon EC2. Figure 5 


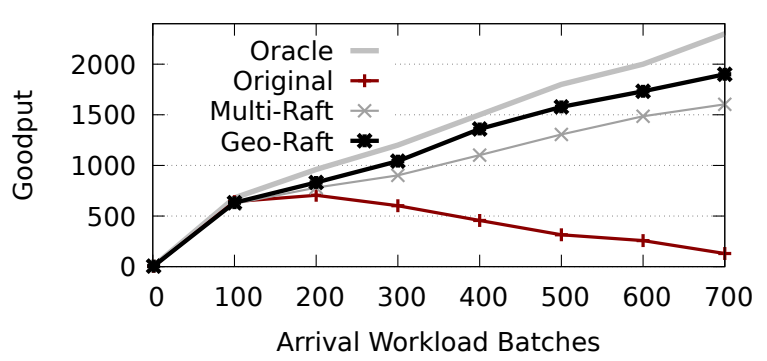

(a) Goodput

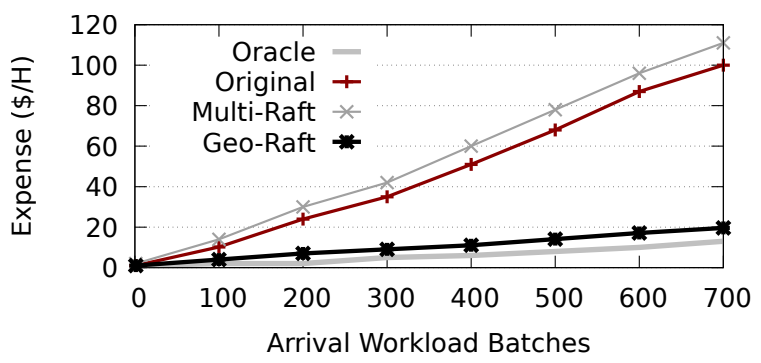

(b) Expense

Figure 6: Performance (a) and cost (b) of Geo-Raft and other baselines at scale-out.

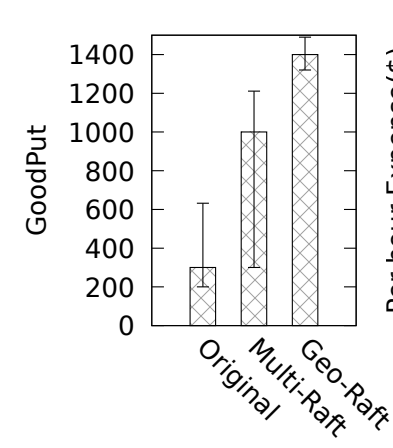

(a) Goodput

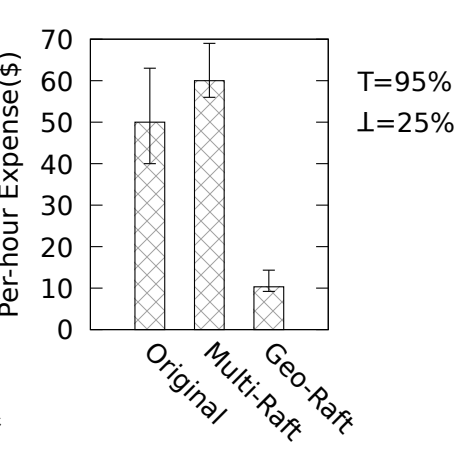

(b) Expense
Figure 7: Performance (a) and cost (b) comparison between Geo-Raft and other baselines.

plots the average latency when executing Read (top) and Write (bottom). For reads, Geo-Raft provides the shortest average response time (i.e., $1.26 \mathrm{~s}$ ), which is $27 \%$ of Multi-Raft (4.6s) and $15 \%$ of Original (7.9s). However, in some extreme cases, Geo-Raft can perform badly, such as overshoots in epoch 410 and 578 in Figure 5(a)top. Similar overshoot happens when Geo-Raft executes larger reads. Such overshoots happen when the majority of observers failed and Geo-Raft had to reschedule workloads to correspondent followers. The overshoot can be mitigated when we spreading observers onto many sites instead of a few cheap ones, which is a tradeoff between performance and cost.

For writes, Geo-Raft scales out in proportion to the ever-increasing updates, nicely. Multi-Raft also scales, however, with a price of $3 \mathrm{X}$ larger response time due to maintaining the 2 pc commit between leaders. Original cannot handle constant updates when scaling-out. When the size of appended logs increases to a certain limit in all nodes, Original fails at the leader blocking the overall write performance, slowing down $2.5 \mathrm{X}$ compared to Geo-Raft. Overall, GeoRaft shows significant performance improvement, as it scales in increments 3-12X compared to Multi-Raft and Original.

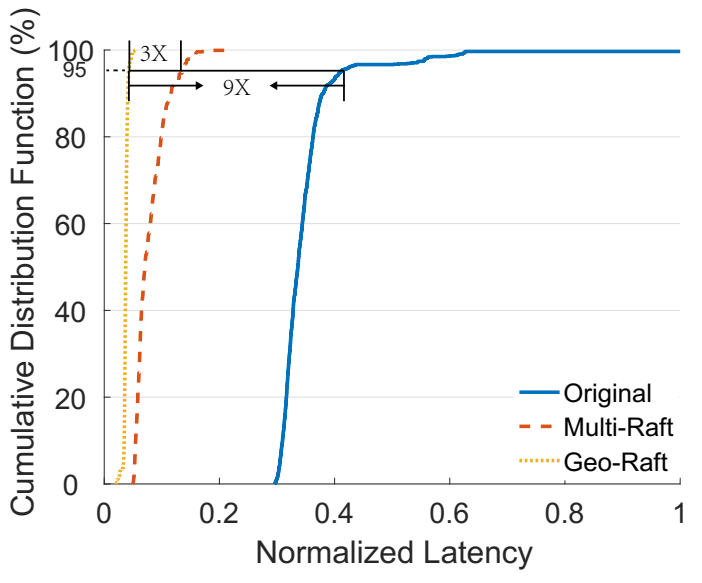

Figure 8: CDF of Geo-Raft and baselines.

Scalability: Geo-Raft can easily scale in proportional to performance and cost. Figure 6 demonstrates such proportionality when the workload batch size increases by 700X. In Figure 6(a), both Geo-Raft and Multi-Raft grow in proportional to the workload size. Geo-Raft exhibits a better scale-out performance than Multi-Raft, and is close to the theoretically best performance in Oracle. Original does not scale. When the workload increases, Original suffers from managing too many logs at the leader node, which prevents scaleout. Multi-Raft can scale out at a much higher cost. As shown in Figure 6(b), Multi-Raft leases more on-demand nodes in scaling out while Geo-Raft harnesses the salient feature of cheap spot instances. Compared to Oracle, Geo-Raft still has rooms to improve, especially in the workload and resource provisioning.

Overall Statistics: Figure 7 shows the overall performance and expense comparison between Geo-Raft and other baselines. In goodput (i.e., correct queried result over unit time), Geo-Raft is $7 \mathrm{X}$ and 1.5X, larger than Original and Multi-Raft, respectively. Considering both reads and writes, Geo-Raft has smaller variation than Multi-Raft. Geo-Raft has significantly smoothed write delay curve due to secretaries often reside in more sites than followers, which reduces unexpected long wide-area network delay. For expenses, Geo-Raft 


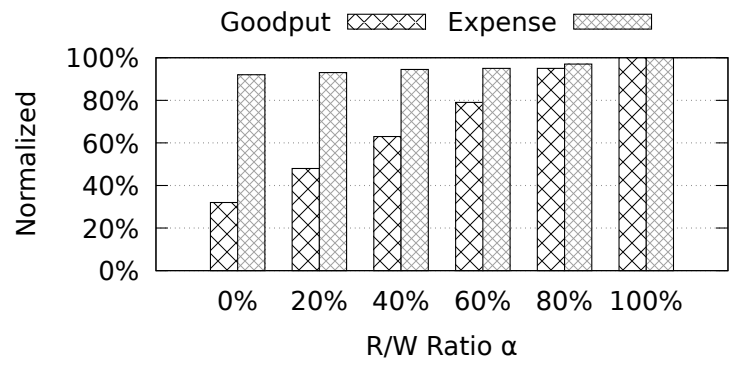

Figure 9: The impact of $\mathrm{R} / \mathrm{W}$ ratio $\alpha$ in Geo-Raft.

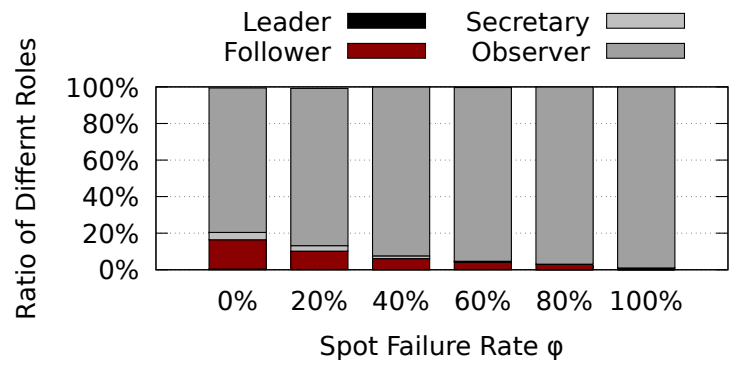

Figure 10: The impact of spot failure rate $\phi$ in Geo-Raft .

exploits cheap spot instances for secretaries and observers in many sites. Geo-Raft spends $86 \%$ and $80 \%$ less than Multi-Raft and Original, respectively. Multi-Raft usually costs more than Original due to its multiple leaders thus expensive resource footprints. Note that, it seems unfair to show this expense comparison while only Geo-Raft can use spot instances. However, there is no existing design on both Raft and Multi-Raft that can exploit spot instances. If we deploy spot instance in Original and Multi-Raft, they can be drown in a nonstop leader re-election and provide barely zero performance.

Performance Distribution. Figure 8 demonstrates the curriculum distribution functions of all jobs running in Geo-Raft and other baselines. A small portion of jobs do suffer in Geo-Raft due to runtime spot instance failures and errors in resource provisioning. In the worst case, Geo-Raft could shrink back as a Raft handling a small number of jobs. However, Geo-Raft has a much shorter tail than Multi-Raft in the latency distribution. Comparing the 95th-percentile SLO, Geo-Raft performs 3X better than Multi-Raft, and 9X better than Original.

Impact of Design Factors: In our provision process, we have two major factors (i.e., workload R/W ratio $\alpha$ and spot instance failure rate $\phi$ ). The factor $\alpha$ affects the provision process in Geo-Raft, while the factor $\phi$ could collapse the provision decision. Figure 9 illustrates the impact of $\alpha$. The average goodput is increasing linearly when Geo-Raft serves more reads than writes. Geo-Raft can handle reads well because Geo-Raft abusively employs many observers to serve

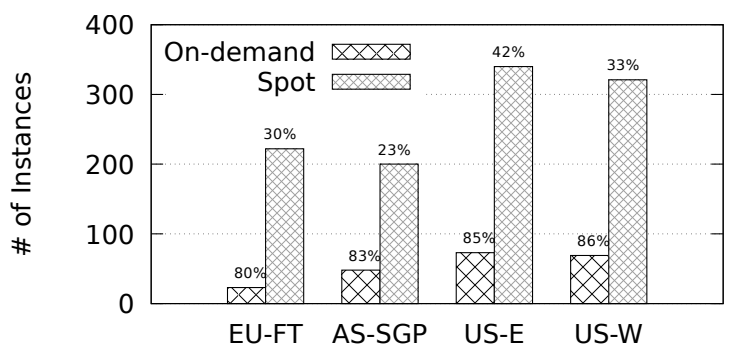

Figure 11: Number of leased instances and average utilizations (on top of the bar) of Geo-Raft in EU-Frankfort, AsiaSingapore, US-East, and US-West.

reads. These observers are cheap, thus the overall expense in GeoRaft grows much slower than performance gain.

Figure 10 shows how spot instances fail affects Geo-Raft. In our model, we assume a static $\phi$ based on history analysis, however, $\phi$ is hard to predict at runtime. When this failure rate increases in a single site, Geo-Raft gradually reduces the number of secretaries at this site, and increases the number of observers in other sites. As such, the number of followers decreases since secretaries decrease. Geo-Raft reduces secretaries on purpose, in order to reduce the possibility of update failure, and thus the cost from the consensus management. While Geo-Raft suffers performance loss from writes, it hedges performance gain from reads as it hires more observers.

Limitations of Geo-Raft in the Wild: We report the server-side statistics in Figure 11. We employ instances from EU-Frankfort, Asia-Singapore, US-East, and US-West. Geo-Raft hires more than 1500 instances, on-demand and spot, in these four sites. As shown in Figure 11, Geo-Raft hires 15-20X more spot instances than ondemand ones. On the one hand, when Geo-Raft leases on-demand instance, it takes the maximum use of the resource, exhibiting more than $80 \%$ utilization in all sites. On the other hand, Geo-Raft abusively leases spot instances without fully exploiting its resources, with an average of $35 \%$ utilization. It is mainly because of the failureprone behavior of spot instances that Geo-Raft only uses a short period during their living period. If the burstable period of spot instance can be accurately predicted, Geo-Raft can provide much better performance at scale-out.

\section{RELATED WORK}

Consensus algorithms, such as Paxos and Raft, are designed to maintain consensus across multiple shared data replicas. These algorithms scales by sharding [28] or automatically adding/dropping followers [12-14, 30]. However, for high write throughput, applications turn to over-provisioned sharding, multiplying inefficiency. Our work, Geo-raft scales incrementally and does so using cheap, failure-prone spot instances.

Raft Consensus Algorithm: In the past few decades, researchers have proposed a large number of consensus algorithms [10, 18, $21,26]$. Raft algorithm [26] is one of the most widely used [2, 7]. Many companies have found that Raft is easy-to-implement and 
provides good performance. Pâris et al. reduced energy footprint of Raft [27]. Gramoli et al. put forward a fast consensus-based dynamic reconfigurations method which can speedup a primary-based rolling upgrade[13]. Copeland et al. propose a Byzantine Fault Tolerant variant of the Raft consensus algorithm [11]. These approaches have not explored efficient scaling out on Raft, while our work focusing on improving the scale-out performance with Raft on spot instances. Geo-Replication: Droopy and Dripple [22] , two sister approaches, reduce latency by dynamically reconfigure leader set. Tuba [9] improves utility by automatic reconfiguration. SPANStore [32] offers low cost storage services making use of the price difference between suppliers. Cadre [33], Lynx [36], and Flutter [17] achieve low latency by avoiding long distance transmission.

Spot Instance Market: In the cloud market, suppliers provide on demand instance and spot instance. Spot instance is usually much cheaper than on-demand instance. However, due to spot instance is unstable and may stop at any moment, it is not reliable for data tasks, especially in maintaining data consistency. There are many research reduce cost by using spot instances. EAIC [19] reduce cost by adaptive checkpointing. And PADB [29] algorithm was put forward get maximized mean profit.

\section{CONCLUSION}

It is challenging to design consensus algorithms that can scale out incrementally and are easy to explain and implement. Raft, a widely implemented algorithm, does not scale well. The Multi-Raft extension can scale out, but inflates costs in geo-distributed settings. We proposed Geo-Raft, a Raft extension that scales in increments 5-7X smaller than Multi-Raft, reducing cost inflation. Geo-Raft transforms log management into a stateless task, allow Raft leaders to offload work to secretaries. Likewise, followers can offload work to observers. Secretaries and observers can be added and removed at runtime to improve throughput or reduce cost. By design, they can exploit low cost but failure-prone spot instances-significantly reducing scale out costs. We prototyped Geo-Raft and plan to release our source code (citation hidden for blind review). Geo-Raft has run continuously on AWS for over 2 months. We have observed that (1) Geo-Raft significantly boosts throughput by up to $9 \mathrm{X}$, compared to Raft and (2) Geo-Raft is $84 \%$ cheaper to deploy than Multi-Raft.

\section{ACKNOWLEDGMENTS}

This research was funded by NSF Grants 1749501 and 135094, and National Science Foundation China (NSFC) grant 617022501006873 , and Jiangxi Natural Science Foundation Youth Grant 91180244.

\section{REFERENCES}

[1] Amazon AWS EC2 Services. https://aws.amazon.com/ec2/.

[2] Cockroach Labs. https://www.cockroachlabs.com.

[3] Google Cluster Data. https://github.com/google/cluster-data.

[4] Open source, containers, and Kubernetes I CoreOS. https://coreos.com.

[5] Oracle I Integrated Cloud Applications and Platform Services. https://www.oracle. com.

[6] Production-Grade Container Orchestration - Kubernete. https://kubernetes.io.

[7] TiDB. https://github.com/pingcap/tidb.

[8] F. Ahmad and T. Vijaykumar. Joint optimization of idle and cooling power in data centers while maintaining response time. In ACM Sigplan Notices, pages 243-256. ACM, 2010.

[9] M. S. Ardekani and D. B. Terry. A Self-Configurable Geo-Replicated Cloud Storage System. OSDI '14, pages 367-381, 2014.
[10] M. Burrows. The chubby lock service for loosely-coupled distributed systems. In Proceedings of the 7 th symposium on Operating systems design and implementation, pages 335-350. USENIX Association, 2006.

[11] C. Copeland and H. Zhong. Tangaroa: a byzantine fault tolerant raft, 2016

[12] N. Deng, C. Stewart, D. Gmach, M. Arlitt, and J. Kelley. Adaptive green hosting. In Proceedings of the 9th international conference on Autonomic computing, pages 135-144. ACM, 2012.

[13] V. Gramoli, L. Bass, A. Fekete, and D. W. Sun. Rollup: Non-disruptive rolling upgrade with fast consensus-based dynamic reconfigurations. IEEE Transactions on Parallel and Distributed Systems, 27(9):2711-2724, 2016.

[14] A. Harlap, A. Chung, A. Tumanov, G. R. Ganger, and P. B. Gibbons. Tributary: spot-dancing for elastic services with latency slos. In 2018 \{USENIX\} Annual Technical Conference ( $\{$ USENIX $\}\{$ ATC $\}$ 18), pages 1-14, 2018.

[15] C.-C. Ho, K. Wang, and Y.-H. Hsu. A fast consensus algorithm for multiple controllers in software-defined networks. In Advanced Communication Technology (ICACT), 2016 18th International Conference on, pages 112-116. IEEE, 2016.

[16] H. Howard, M. Schwarzkopf, A. Madhavapeddy, and J. Crowcroft. Raft Refloated. ACM SIGOPS Operating Systems Review, 49(1):12-21, 2015.

[17] Z. Hu, B. Li, and J. Luo. Time-and cost-efficient task scheduling across geodistributed data centers. IEEE Transactions on Parallel and Distributed Systems, 29(3):705-718, 2018.

[18] P. Hunt, M. Konar, F. P. Junqueira, and B. Reed. Zookeeper: Wait-free coordination for internet-scale systems. In USENIX annual technical conference, volume 8, page 9. Boston, MA, USA, 2010.

[19] I. Jangjaimon and N.-F. Tzeng. Effective cost reduction for elastic clouds under spot instance pricing through adaptive checkpointing. IEEE Transactions on Computers, 64(2):396-409, 2015.

[20] N. K. Jong and P. Stone. State abstraction discovery from irrelevant state variables. In IJCAI, volume 8, pages 752-757, 2005 .

[21] L. Lamport et al. Paxos made simple. ACM Sigact News, 32(4):18-25, 2001.

[22] S. Liu and M. Vukolić. Leader set selection for low-latency geo-replicated state machine. IEEE Transactions on Parallel and Distributed Systems, 28(7):19331946, 2017.

[23] N. Morris, C. Stewart, L. Chen, R. Birke, and J. Kelley. Model-driven computational sprinting. In Proceedings of the Thirteenth EuroSys Conference, page 38. ACM, 2018.

[24] Y. Niu, F. Liu, X. Fei, and B. Li. Handling flash deals with soft guarantee in hybrid cloud. In IEEE INFOCOM 2017-IEEE Conference on Computer Communications, pages 1-9. IEEE, 2017.

[25] Y. Niu, B. Luo, F. Liu, J. Liu, and B. Li. When hybrid cloud meets flash crowd: Towards cost-effective service provisioning. In 2015 IEEE Conference on Computer Communications (INFOCOM), pages 1044-1052. IEEE, 2015.

[26] D. Ongaro and J. K. Ousterhout. In search of an understandable consensus algorithm. In USENIX Annual Technical Conference, pages 305-319, 2014.

[27] J.-F. Pâris and D. D. Long. Reducing the energy footprint of a distributed consensus algorithm. In Dependable Computing Conference (EDCC), 2015 Eleventh European, pages 198-204. IEEE, 2015.

[28] J. Rao, E. J. Shekita, and S. Tata. Using paxos to build a scalable, consistent, and highly available datastore. Proceedings of the VLDB Endowment, 4(4):243-254, 2011.

[29] Y. Song, M. Zafer, and K. W. Lee. Optimal bidding in spot instance market. In Proceedings - IEEE INFOCOM, pages 190-198, 2012.

[30] C. Wang, B. Urgaonkar, G. Kesidis, A. Gupta, L. Y. Chen, and R. Birke. Effective capacity modulation as an explicit control knob for public cloud profitability. ACM Transactions on Autonomous and Adaptive Systems (TAAS), 13(1):2, 2018.

[31] D. Woos, J. R. Wilcox, S. Anton, Z. Tatlock, M. D. Ernst, and T. Anderson. Planning for change in a formal verification of the raft consensus protocol. In Proceedings of the 5th ACM SIGPLAN Conference on Certified Programs and Proofs, pages 154-165. ACM, 2016.

[32] Z. Wu, M. Butkiewicz, D. Perkins, E. Katz-Bassett, and H. V. Madhyastha. SPANStore: cost-effective geo-replicated storage spanning multiple cloud services. SOSP'13, pages 292-308, 2013.

[33] Z. Xu, N. Deng, C. Stewart, and X. Wang. Cadre: Carbon-aware data replication for geo-diverse services. In 2015 IEEE International Conference on Autonomic Computing, pages 177-186. IEEE, 2015.

[34] Z. Xu, C. Stewart, N. Deng, and X. Wang. Blending on-demand and spot instances to lower costs for in-memory storage. In INFOCOM 2016-The 35th Annual IEEE International Conference on Computer Communications, IEEE, pages 1-9. IEEE, 2016.

[35] X. Yi, F. Liu, Z. Li, and H. Jin. Flexible instance: Meeting deadlines of delay tolerant jobs in the cloud with dynamic pricing. In 2016 IEEE 36th International Conference on Distributed Computing Systems (ICDCS), pages 415-424. IEEE, 2016.

[36] Y. Zhang, R. Power, S. Zhou, Y. Sovran, M. K. Aguilera, and J. Li. Transaction chains: achieving serializability with low latency in geo-distributed storage systems. SOSP '13, pages 276-291, 2013. 